\title{
CONCERNING ACCESSIBILITY IN THE PLANE AND REGULAR ACCESSIBILITY IN $n$ DIMENSIONS*
}

\author{
BY G. T. WHYBURN
}

1. Accessibility in the Plane. The point set $K$ is said to be everywhere accessible from a point set $R$ provided that if $A$ and $B$ are any two points belonging to $K$ and $R$ respectively, then there exists a simple continuous arc $A B$ from $A$ to $B$ such that $A B-A$ is a subset of $R$. In my paper Concerning plane closed point sets which are accessible from certain subsets of their complements, $\dagger$ among other results, I proved the following theorem.

Theorem A. If, in a plane $S, K$ is a point set such that there exist three mutually exclusive connected subsets $R_{1}, R_{2}$, and $R_{3}$ of $S-K$ such that $K$ is everywhere accessible from $R_{1}$ and $R_{2}$ and every point of $K$ is a limit point of $R_{3}$, then $K$ contains not more than two points.

In this section it is purposed to establish some results which are related to Theorem $\mathrm{A}$ and in the proof of which use will be made of Theorem A. It is obvious from Theorem A that there does not exist any plane continuum, which is everywhere accessible from each one of a particular group of three of its complementary domains. It is not so apparent, however, that there does not exist a plane continuum each point of which is accessible from each one of some group of three of the complementary domains of this continuum. This result will be demonstrated in this present paper as an immediate consequence of a somewhat more general theorem. It will also be shown that if each point of a bounded plane continuum $M$ is accessible from each of two complementary

* Presented to the Society, December 28, 1927.

$\dagger$ Offered to the Proceedings of the National Academy of Sciences. See Theorem 1, and remark at the end of proof of Theorem 1. 
domains of $M$, then $M$ is a Menger regular curve* which has at most a countable number of cut points.

THEOREM 1. If $G$ is any countable collection of mutually exclusive connected point sets in the plane $S, T$ denotes the sum of all the point sets of the collection $G$, and $K$ denotes the set of all points $X$ in $S-T$ such that $X$ is accessible from at least three of the sets of $G$, then $K$ is countable.

Proof. $\dagger$ Suppose, on the contrary, that $K$ is uncountable. Then since $G$ is countable and each point of $K$ is accessible from at least one set of the collection $G$, it follows that there exists a set $R_{1}$ of the collection $G$ such that each point of an uncountable subset $K_{1}$ of $K$ is accessible from $R_{1}$. Since $K_{1}$ is uncountable and the collection $G_{1}$ obtained by omitting $R_{1}$ from the collection $G$ is countable, and since each point of $K$ is accessible from at least one set of $G_{1}$, it follows that there exists a set $R_{2}$ of the collection $G_{1}$ such that each point of an uncountable subset $K_{2}$ of $K_{1}$ is accessible from $R_{2}$. Let $G_{2}$ be the collection obtained by omitting the set $R_{2}$ from the collection $G_{1}$. Then since $K_{2}$ is uncountable and $G_{2}$ is countable, and each point of $K_{2}$ is accessible from at least one set of the collection $G_{2}$, there exists a set $R_{3}$ of the collection $G_{2}$ such that each point of an uncountable subset $K_{3}$ of $K_{2}$ is accessible from $R_{3}$. But then every point of $K_{3}$ is accessible from each of the three mutually exclusive connected subsets $R_{1}, R_{2}$, and $R_{3}$ of $S-K_{3}$, and hence, by Theorem A, $K_{3}$ can contain at most two points. But $K_{3}$ is uncountable. Thus the supposition that $K$ is uncountable leads to a contradiction; and the truth of Theorem 1 is therefore established.

Since the complementary domains of every plane continu-

* See the definition of this term below.

$\dagger$ Professor W. A. Wilson has kindly called my attention to the following very concise proof of Theorem 1 : Since $G$ is countable, the number of groups of three sets of $G$ is countable. It follows from Theorem A that at most two points of $K$ are accessible from each set of any one of these groups. Hence the set of points of $K$ must be countable. 
um are countable, we have the following immediate corollaries.

Corollary 1. If $M$ is any plane continuum, then not more than a countable number of points of $M$ are accessible from each of three complementary domains of $M$.

Corollary 2. There does not exist, in the plane, a continuum $M$ every point of which is accessible from each of three distinct complementary domains of $M$.

Definition. A continuum $M$ is said to be a Menger regular curve* provided that if $P$ is any point of $M$ and $\epsilon$ is any positive number, then there exists a connected open subset $R$ of $M$ which contains $P$, is of diameter $<\epsilon$, and whose $M$-boundary is finite. The $M$-boundary of an open subset $R$ of a point set $M$ is the set of all those points of $M-R$ which are limit points of $R$.

THEOREM 2. If every point of a plane continuum $M$ is accessible from at least two of the complementary domains of $M$ then $M$ is a Menger regular curve; and if $M$ is bounded, then it has not more than a countable number of cut points.

Proof. I shall first show that $M$ is a Menger regular curve. Suppose, on the contrary, that $M$ is not a Menger regular curve. Then by a theorem of Menger's $\dagger$ there exists a subcontinuum $H$ of $M$ no point of which is a regular point of $M$. Now let $D_{1}, D_{2}, D_{3}, \cdots$ be the complementary domains of $M$. For each $i$, let $B_{i}$ denote the boundary of $D_{i}$, and let $A_{i}$ denote the set of points $H \cdot B_{i}$. Then, for each $i$, $A_{i}$ is a closed point set. Since, by hypothesis, every point of $H$ is accessible from one of the domains $D_{1}, D_{2}, \cdots$, it follows that $H=A_{1}+A_{2}+A_{3}+\cdots$. It is well known that no continuum is the sum of a countable number of closed point sets each of which is totally disconnected. Therefore, for some integer $p, A_{p}$ contains a continuum $K$. Now for each integer $i \neq p$, let $C_{i}$ denote the set of points $K \cdot B_{i}$. Then

* See K. Menger, Grundzige einer Theorie der Kurven, Mathematische Annalen, vol. 95 (1925), pp. 277-306.

$\dagger$ See K. Menger, loc. cit., p. 288, Theorem 8. 
since, by hypothesis, every point of $K$ is accessible from at least one of the domains $D_{1}, D_{2}, \cdots, D_{p-1}, D_{p+1}, \cdots$, it follows that $K=C_{1}+C_{2}+\cdots+C_{p-1}+C_{p+1}+\cdots$. And since, for each $i, C_{i}$ is closed, it follows just as above that there exists an integer $q \neq p$ such that $C_{q}$ contains a bounded continuum $T$. The continuum $T$ belongs to the boundary of each of the domains $D_{p}$ and $D_{q}$.

Now all, save possibly a countable number, of the points of $T$ must be accessible from $D_{p}$. For if on the contrary, an uncountable subset $Q$ of $T$ exists, no point of which is accessible from $D_{p}$, then since, by hypothesis, every point of $T$ is accessible from at least two complementary domains of $M$, it follows by an argument similar to that given in the proof of Theorem 1 that there exists an uncountable subset $W$ of $Q$ and two integers $j$ and $k$ each distinct from $p$, and such that each point of $W$ is accessible from each of the domains $D_{j}$ and $D_{k}$. But since every point of $W$ is a limit point of $D_{p}$, this contradicts Theorem A. Hence all, save possibly a countable number, of the points of $T$ are accessible from $D_{p}$. Likewise all, save possibly a countable number of the points of $T$, are accessible from $D_{q}$. Therefore there exists a countable, or vacuous, subset $U$ of $T$ such that every point of $T-U$ is accessible from each of the domains $D_{p}$ and $D_{q}$.

Let $X$ and $Y$ be two points of $T-U$. Then since $X$ and $Y$ are accessible from each of the domains $D_{p}$ and $D_{q}$, it readily follows that there exist points $P$ and $Q$ in $D_{p}$ and $D_{q}$ respectively and arcs $P X Q$ and $P Y Q$ from $P$ to $Q$ which have in common only the points $P$ and $Q$, and such that the point set $P X+P Y-(X+Y)$ is a subset of $D_{p}$ and the set $Q X+Q Y$ $-(X+Y)$ is a subset of $D_{q}$. Let $J$ denote the simple closed curve $P X Q Y P$. Then since $T$ is bounded and has only the points $X$ and $Y$ in common with $J$, it follows by a theorem of Rosenthal's* that either the interior or the exterior of $J$

* A. Rosenthal, Teilung der Ebene durch irreduzible Kontinua, Sitzungsberichte der Mathematisch-Physikalischen Klasse der Bayerischen Akademie der Wissenschaften zu München, 1919, p. 104. 
contains a connected subset $g$ of $T$ such that each of the points $X$ and $Y$ is a limit point of $g$. The two cases are practically alike, so let us suppose that $g$ is a subset of the interior of $J$. Let $G$ denote the subset of $M$ which lies within $J$, and let $N$ denote the set of points $G+X+Y$. Then since $g+X+Y$ is connected it follows that $N$ is a continuum.

Let $E$ denote the set of all those points of $T-U$ which belong to $g$. Then since $U$ is countable, and $g$ is a connected subset of $T$, it follows that $E$ is uncountable. Let $Z$ be any point of $E$. Then since $Z$ is accessible from each of the domains $D_{p}$ and $D_{q}$, it readily follows that there exists an $\operatorname{arc} A Z B$, where $A$ is a point on the $\operatorname{arc} X P Y$ of $J$ and $B$ a point on the $\operatorname{arc} X Q Y$ of $J$, and such that $A Z-Z$ is a subset of $D_{p}$ and $B Z-Z$ is a subset $D_{q}$. The arc $A Z B$ divides the interior of $J$ into two regions $R_{x}$ and $R_{y}$ whose boundaries contain $X$ and $Y$ respectively. And if $S_{x}$ denotes the set of points $N \cdot R_{x}+X$ and $S_{y}$ the set of points $N \cdot R_{y}+Y$, then it is clear that $S_{x}$ and $S_{y}$ are mutually separated sets, and that $S_{x}+S_{y}=N-Z$. Therefore each point $Z$ of $E$ is a cut point of $N$. But since $E$ is uncountable, then by a theorem of the author* it follows that $E$ contains at least one point $V$ which is a regular point (in fact a point of Menger order two) of $N$; and since $M-N$ contains no point within $J$, it follows that $V$ is a regular point of $M$. But $V$ belongs to $H$, and by supposition no point of $H$ is a regular point of $M$. Thus the supposition that $M$ is not a Menger regular curve leads to a contradiction.

Now suppose $M$ is bounded, and that, contrary to the second part of this theorem, it has uncountably many cut points. Then by the above quoted theorem of the author, at least one of these cut points of $M$ must be a point of Menger order two of $M$. But by another theorem of the author $\dagger$ a cut point of Menger order two of a bounded

${ }^{*}$ G. T. Whyburn, Concerning the cut points of continua, Transactions of this Society, vol. 30 (1928), No. 3. The theorem used here states that all, save possibly a countable number, of the cut points of a plane continuum $M$ are points of Menger order two of that continuum.

$\dagger$ Loc. cit., Theorem 10. 
continuum $M$ can belong to the boundary of at most one complementary domain of $M$; and by our hypothesis every point of $M$ must belong to the boundaries of at least two complementary domains of $M$. Thus the supposition that $M$ has uncountably many cut points leads to a contradiction, and the theorem is proved.

It is of interest to note that every bounded continuum satisfying the hypothesis of Theorem 2 is a type of continuous curve which has elsewhere* been called a two-way continuous curve, that is, every two of its points can be joined by two different arcs which lie wholly in the curve.

2. Regular Accessibility in $n$ Dimensions. A limit point $P$ of a point set $R$ is said to be regularly accessible $\nmid$ from $R$ provided that if $\epsilon$ is any positive number, then there exists a $\delta_{e p}>0$ such that every point $X$ of $R$ whose distance from $P$ is $<\delta_{\epsilon p}$ can be joined to $P$ by an arc $X P$ of diameter $<\epsilon$ such that $X P-P$ is a subset of $R$.

THEOREM 3. In order that the boundary point $P$ of a connected open subset $\$ R$ of a continuous curve $M$ in a euclidean space of $n$ dimensions should be regularly accessible from $R$, it is necessary and sufficient that the point set $R+P$ should be connected im kleinen at $P$.

Proof. That the condition is necessary is an immediate consequence of the definition of regular accessibility. I shall show that the condition is sufficient. The case where $P$ belongs to $R$ is evident, so let us suppose that $P$ belongs to $M-R$. Let $\epsilon$ be any number $>0$. By hypothesis there exists a $\delta_{\epsilon p}>0$ such that every point $X$ of $R$ at a distance $<\delta_{\epsilon p}$ from $P$ can be joined to $P$ by a connected subset of $R+P$ of diameter $<\epsilon / 2$. Let $G$ denote the set of all those

* See G. T. Whyburn, Two-way continuous curves, this Bulletin, vol. 32 (1926), pp. 659-663.

† See G. T. Whyburn, Concerning the open subsets of a plane continuous curve, Proceedings of the National Academy, vol. 13 (1927), pp. 650-657.

$\ddagger$ The subset $R$ of a closed point set $M$ is an open subset of $M$ provided that $M-R$ is either vacuous or closed. 
points of $R$ whose distance from $P$ is $\left\langle\delta_{\epsilon p}\right.$, let $X_{1}$ be any point of $G$, and let $R_{1}$ denote the maximal connected subset of the set of all those points of $R$ whose distance from $P$ is $<\epsilon / 2$ which contains $X_{1}$. Then $R_{1}$ is a connected open subset of $M$ which is of diameter $<\epsilon$; furthermore, $P$ is a limit point of $R_{1}$. For suppose it is not. By hypothesis a connected subset $N$ of $R+P$ exists which contains both $P$ and $X_{1}$ and is of diameter $<\epsilon / 2$. Since $N$ is connected and contains the point $X_{1}$ of $R_{1}$ and the point $P$ which is neither a point nor a limit point of $R_{1}$, it therefore contains a point $Q$, distinct from $P$, which belongs to $R-R_{1}$ and is a limit point of $R_{1}$. But $Q$ is at a distance $<\epsilon / 2$ from $P$, and by definition $R_{1}$ is a maximal connected subset of the set of all points of $R$ a distance $<\epsilon / 2$ from $P$. Thus the supposition that $P$ is not a limit point of $R_{1}$ leads to a contradiction. Hence $P$ is a limit point of $R_{1}$. In a similar way it follows that there exists a connected open subset $R_{2}$ of $M$ which is a subset of $R_{1}$, is of diameter $<\epsilon / 2$, and has $P$ for a limit point; and so on. In general, for each positive integer $n$, a connected open subset $R_{n}$ of $M$ exists which is a subset of $R_{n-1}$, is of diameter $<\epsilon / n$, and has $P$ for a limit point. For each $n$, the set $R_{n}$ contains a point $X_{n}$ and an $\operatorname{arc}^{*} X_{n} X_{n+1}$. It is easy to see that the point set $P+X_{1} X_{2}+X_{2} X_{3}+\cdots$ $+X_{n} X_{n+1}+\cdots$ is closed and that it contains an $\operatorname{arc} X_{1} P$ of diameter $<\epsilon$ such that $X_{1} P-P$ is a subset of $R$. And since $X_{1}$ is any point of $G$, it follows that $P$ is regularly accessible from $R$.

Corollary. In order that a boundary point $P$ of a domain $D$, in n-dimensional space, should be regularly accessible from $D$ it is necessary and sufficient that the set of points $D+P$ should be connected im kleinen.

The University of TeXas

* See R. L. Moore, Mathematische Zeitschrift, vol. 15 (1922), Theorem 